\title{
All-optical regeneration based on phase sensitive amplification
}

\author{
Radan Slavík*, Joseph Kakande, Francesca Parmigiani, Periklis Petropoulos, and David J. Richardson \\ Optoelectronics Research Centre, University of Southampton, Southampton, SO17 1BJ, UK \\ *r.slavik@soton.ac.uk
}

\begin{abstract}
We review recent results regarding regeneration of binary and quadruple phase encoded signals using phase sensitive amplification in fibers.

(C)2011 Optical Society of America

OCIS codes: (060.2320) Fiber optics amplifiers and oscillators, (190.4410) Nonlinear optics, parametric processes
\end{abstract}

\begin{abstract}
1. Introduction
Phase noise introduced during transmission both from optical amplifiers and the nonlinear interactions between channels represents a significant limiting factor to data transmission using (differential) phase-shift keyed, modulation formats [1]. Consequently, there is great interest in developing all-optical regeneration schemes capable of eliminating phase (and ideally additionally amplitude) noise for phase shift keyed signals. Phase regeneration of binary-encoded signals (PSK, DPSK) can be implemented directly by exploiting the phase-squeezing capability of phase sensitive amplifiers (PSAs) [2]. To perform also amplitude regeneration, PSA is operated in saturation regime. To achieve PSA, however, a phase relationship between the PSA pump(s), the signal and any idlers present needs to be maintained. Recently, a scheme in which the carrier from a carrier-less PSK signal is recovered and then used to phase-lock the locally generated pumps with the incoming data prior to a degenerate PSA was published [3].

For regeneration of more complex modulation formats, e.g., quadruple phase-shift keyed (QPSK) signals, more complex PSA configurations have been proposed and very recently demonstrated [4]. Generally they include cascaded/simultaneous four wave mixing processes to achieve regeneration of more than two phase levels that are achieved in a simple PSA.

In the talk, we will present detailed analysis and discuss practical aspects associated with a PSK regenerator with just a noisy carrier-less data at its input. Further, we shall explain schemes for the regeneration of more complex modulation formats.
\end{abstract}

\section{PSK regenerator}

The most practical implementation of a PSK regenerator demonstrated so far [5] is shown in Fig. 1. The non-returnto-zero (NRZ)-DPSK data was sent through a phase modulator to add emulated noise. The distorted signal was then launched into the regenerator which was made of polarisation maintaining (PM) components other than the part operating in reflection (where the polarization was inherently preserved as a result of the bidirectional propagation) and the highly nonlinear fiber (HNLF) used for PSA (HNLF 2). First, the signal was combined with a CW local pump ('Pump 1', with a $200 \mathrm{GHz}$ offset from the data carrier frequency) using an add-multiplexer (data insertion loss $\sim 0.5 \mathrm{~dB}$ ) and then sent to a PM-HNLF (HNLF 1) to generate an idler wave that was inherently phase-locked to the data and Pump 1. Note that due to the phase erasure process, the binary data modulation was not transferred to the idler [3]. Then, the three signals (Data, Pump 1, Idler) were demultiplexed in a 4-channel 200-GHz demux (insertion loss $\sim 1 \mathrm{~dB}$ ) placed behind a circulator. A mirror provided retro-reflection in the data path as well in the path for Pump 1 and which also included a PZT stretcher and a variable attenuator. A semiconductor laser [6], which was injection locked to the idler, was used to generate Pump 2 in the idler path. Pump 2 was thus phase-locked to the idler (and thus also to the data and Pump 1). Note that since the injection locking is a much slower process than four wave mixing (having a (sub)-GHz bandwidth) then any high frequency fluctuations (e.g., bit-to-bit phase variations) present on the original data signal are not transferred onto the output of the slave laser [3]. Note that all the optical waves involved in the regeneration process share a common path through the regenerator other than on the output side of the demux. The data stream with the two properly locked pumps was then launched into the PSA which consisted of a high-power EDFA (total power of $29 \mathrm{dBm}$ ) and a 180-m sample of a high-SBS-threshold HNLF [7]. For optimum performance the PSA was operated in deep saturation, resulting in a strong variation of pump power at the PSA output as a function of the relative phase between the pump and signal beams. We used this feature as an error signal to control a PZT fiber stretcher in order to compensate for phase drifts due to environmental changes (temperature). 


\section{CWD2.pdf}

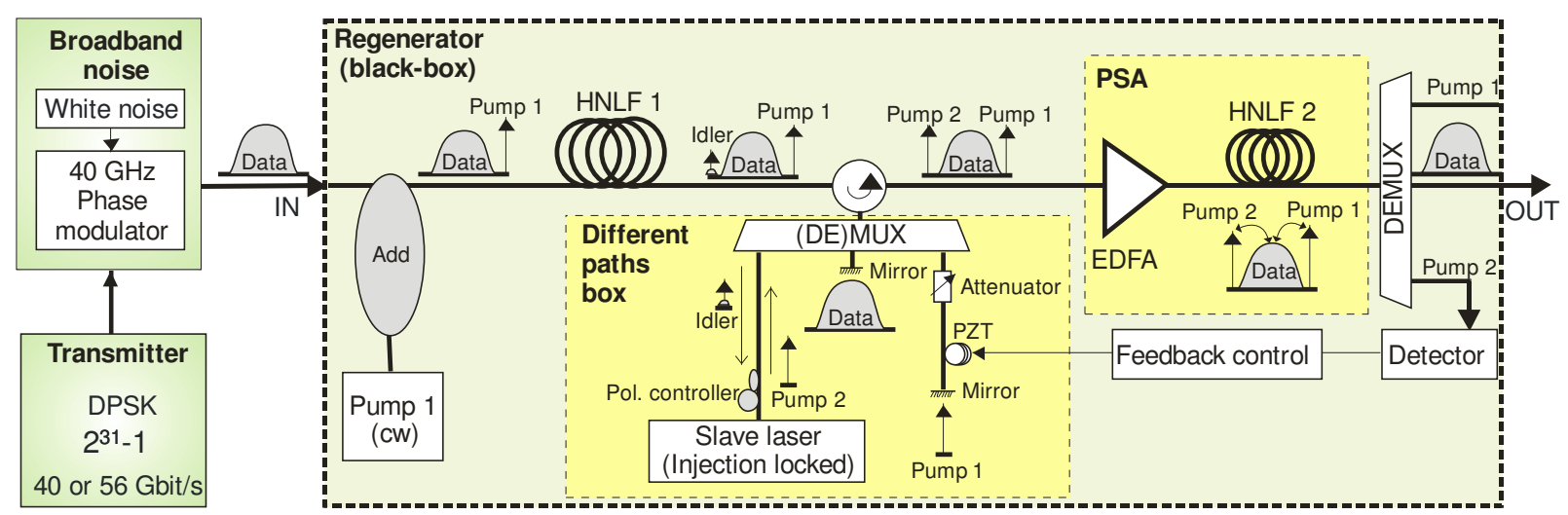

Figure 1: Experimental set-up for the PSK regenerator.

\section{QPSK regenerator}

Multi-level phase regeneration requires a stair-case phase transfer function. In [4] it was proposed to achieve this form of response by interfering the signal with a conjugated $(\mathrm{M}-1)^{\text {th }}$ phase harmonic, where $\mathrm{M}$ is number of phase levels used. For example, for QPSK $(\mathrm{M}=4)$ signal, phase harmonics of the signal were generated using four wave mixing with a CW pump first, Fig. 2 . The $2^{\text {nd }}$ harmonics was subsequently dropped and the $4^{\text {th }}$ harmonics which is CW (as any phase level presented in QPSK of 0, 90, 180, 270 deg multiplied by four gives zero phase level) was used for phase locking of a second pump in the following 2-pump non-degenerate PSA, Fig. 2. By analyzing the phase condition of this PSA it is found that signals of phases of 0,90,180, 270 deg are amplified, while signals of phases of 45, 135, 225, $315 \mathrm{deg}$ are de-amplified. This is exactly what is needed for QPSK regeneration. Reconfiguring the regenerator to an alternate modulation format, such as 8-PSK, can be achieved simply by generating a broader FWM comb and selecting the desired harmonics.

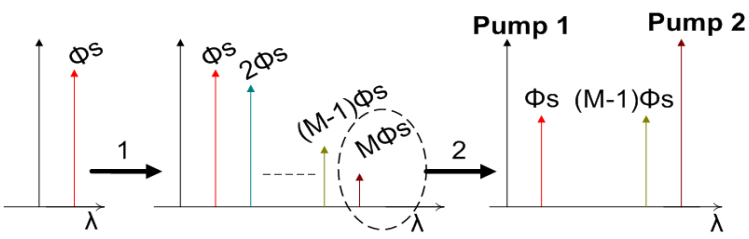

Fig. 2: Multi-level regeneration in two steps: (1) Nonlinear generation of phase harmonics via four wave mixing followed by pump recovery, (2) Parametric 2-pump non-degenerate phase sensitive amplification.

\section{Conclusions}

Phase sensitive amplification allows for the regeneration of phase encoded signals without the need for phase locked signals to be transmitted through the fiber link and is not limited to binary encoded signals. However, further work on the regeneration of wavelength multiplexed signals (WDM), which has not been so far demonstrated, will be needed if the technology is ever to become practical.

\section{References}

[1] J. P. Gordon, and L. F. Mollenauer, "Phase noise in photonic communications systems using linear amplifiers," Opt. Lett. 15(23), 1351-1353 (1990).

[2] K. Croussore, et al., "Demonstration of phase-regeneration of DPSK signals based on phase-sensitive amplification," Opt. Express, 13, 39453950 (2005).

[3] R. Slavík et al, "All-optical phase and amplitude regenerator for next-generation telecommunications systems," Nature Photonics 4 , 690 695 (2010).

[4] J. Kakande et al, "First demonstration of all-optical QPSK signal regeneration in a novel multi-format phase sensitive amplifier," ECOC 2010 Turin 19-23, Italy, PD 3.3, Sep 2010.

[5] R. Slavík et al, "Robust design of all-optical PSK regenerator based on phase sensitive amplification, " submitted to OFC 2011.

[6] R. Phelan, B. Kelly, J. O'Carroll, C. Herbert, A. Duke and J. O'Gorman, "- $40^{\circ} \mathrm{C}<\mathrm{T}<95^{\circ} \mathrm{C}$ mode-hop free operation of an uncooled AlGaInAs-MQW discrete-mode laser diode with emission at $\lambda=1.3 \mu \mathrm{m}$.” Electron. Lett. 45, 43-45 (2009).

[7] L. Grüner-Nielsen et al., "A Silica Based Highly Nonlinear Fibre with Improved Threshold for Stimulated Brillouin Scattering," paper Tu.4.D.3, ECOC 2010. 